\title{
Exponential trichotomies and continuity of invariant manifolds
}

\section{Severino Horácio da Silva*}

Unidade Acadêmica de Matemática e Estatística UAME/CCT/UFCG,

Rua Aprígio Veloso, 882, Bairro Universitário CEP 58429-900, Campina Grande-PB, Brasil

E-mail address: horaciousp@gmail.com and horacio@dme.ufcg.edu.br

\section{Antonio Luiz Pereira ${ }^{\dagger}$}

Instituto de Matemática e Estatística, Universidade de São Paulo, Rua do Matão 1010, Cidade Universitária, CEP 05508-090, São Paulo-SP, Brasil

E-mail address: alpereir@ime.usp.br

\begin{abstract}
In this work, we consider the invariant manifolds for the family of equations

$$
\dot{x}=A x+f(\varepsilon, x),
$$

where $A$ the is generator of a strongly continuous semigroup of linear operators in a Banach space $X$ and $f(\varepsilon, \cdot): X \rightarrow X$ is continuous. The existence of stable (unstable) and center-stable (center-unstable) manifolds for a large class of these equations has been proved in [2]. We prove here that, if $A$ admits a exponential trichotomy and $f$ satisfies some suitable regularity hypotheses, then those manifolds are continuous with respect to the parameter $\varepsilon$.
\end{abstract}

\section{Introduction}

There exists a large literature on the existence and properties of stable (unstable) and center-stable (center-unstable) invariant manifolds for the problem

$$
\dot{x}=A x+f(x),
$$

2000 Mathematics Subject Classification. 34K19; 34D09; 34G20.

Key words: Exponential trichotomy; Invariant manifolds; Continuity of manifolds.

*Partially supported by CNPq-Brazil grants 141882/2003-4, 620150/2008-4 from Casadinho and 5733523/2008-8 from INCTMat.

${ }^{\dagger}$ Partially supported by CNPq-Brazil grants 2003/11021-7, 03/10042-0. 
under various assumptions.

We may cite for example, [1], [2], [5], [6], [7], [8], [10], [11], [13], [14], [15], [16] and [17]. The continuity of these sets with respect to parameters has also been investigated, assuming (uniform) exponential dicothomy for the operator $A$ in [1], [10], [8] and [16], for instance. Existence and continuity, assuming nonuniform exponential dichotomy has been proved in [3] and [4]. For the case of exponential trichotomy, the continuity of these sets was proved in [5] and [14]. However, [14] treats only discrete systems and [5], as well as [14], use normal hyperbolicity.

As far as we know, the continuous case, without assuming normal hyperbolicity, has been considered for the first time in the first author's PhD's thesis (in Portuguese, see [18]). It is our aim here to present, among others, the result obtained there for this problem.

To be precise, we state below the hypotheses used throughout, and fix some notation, which are basically the same of [2].

Let $X$ be a Banach space with norm $|\cdot|, A: D(A) \subset X \rightarrow X$ the generator of a strongly continuous semigroup of linear operators $\{T(t)\}_{t \geq 0}$ on $X$ and suppose $f: X \rightarrow X$ is a continuous function satisfying

(H1) $f(0)=0$,

(H2) $\| f(\varphi)-f(\psi))\|\leq \eta(r)\| \varphi-\psi\|,\| \varphi\|,\| \psi \|<r$,

where $\eta$ is non decreasing continuous function to real values on $[0, \infty)$ with $\eta(0)=0$.

We also assume the following hypotheses for the semigroup.

(H3) (BU - Backwards Uniqueness). For each $t \geq 0, T(t)$ is injective;

(H4) $X$ admits the following decomposition:

(H4a) $X=\pi_{-} X \oplus \pi_{0} X \oplus \pi_{+} X$, where $\pi_{-}, \pi_{0}, \pi_{+}$are continuous linear projections on $X$.

The condition (H4a) implies

$$
\pi_{-} \pi_{0}=\pi_{0} \pi_{-}=\pi_{-} \pi_{+}=\pi_{+} \pi_{-}=\pi_{+} \pi_{0}=\pi_{0} \pi_{+}=0,
$$

and

$$
\pi_{-} \pi_{-}=\pi_{-}, \pi_{0} \pi_{0}=\pi_{0}, \pi_{+} \pi_{+}, \pi_{-}+\pi_{0}+\pi_{+}=I .
$$

If $\varphi \in X$, we write $\varphi_{-}=\pi_{-} \varphi, \varphi_{0}=\pi_{0} \varphi, \varphi_{+}=\pi_{+} \varphi$.

Throughout we shall use the equivalent norm $\|\cdot\|$ on $X$, where, for each $\varphi \in X$

$$
\|\varphi\|=\left|\varphi_{-}\right|+\left|\varphi_{0}\right|+\left|\varphi_{+}\right|
$$


(H4b) For each $t \geq 0, T(t)$ commutes with the operators $\pi_{-}, \pi_{0}, \pi_{+}$so that each of the subspaces $\pi_{-} X, \pi_{0} X, \pi_{+} X$ is invariant under $T(t)$. Furthermore, $T(t)$ may be extended to a continuous group of linear operators on $\pi_{0} X \oplus \pi_{+} X$;

(H4c) There exist constants

$$
a_{-}, a_{0}, a_{+}, \min \left\{a_{-}, a_{+}\right\}>a_{0} \geq 0 \text { and } K>1 ;
$$

(without loss of generality we assume $a_{0}>0$ ), such that

(H4c.i) $\quad\left\|T(t) \varphi_{-}\right\| \leq K e^{-a_{-} t}\left\|\varphi_{-}\right\|, \forall \varphi \in X, t \geq 0$;

(H4c.ii) $\left\|T(t) \varphi_{0}\right\| \leq K e^{a_{0}|t|}\left\|\varphi_{0}\right\|, \forall \varphi \in X, t \in \mathbb{R}$;

(H4c.iii) $\left\|T(t) \varphi_{+}\right\| \leq K e^{a_{+} t}\left\|\varphi_{+}\right\|, \forall \varphi \in X, t \leq 0$.

It is well known (see for example [9] and [13]) that, if $f$ satisfies (H1) and (H2), then the Cauchy problem

$$
\begin{aligned}
\dot{x} & =A x+f(x) \\
x(0) & =x_{0} .
\end{aligned}
$$

has a unique local 'mild solution', that is, a solution of the integral equation

$$
w(t)=T(t) w(0)+\int_{0}^{t} T(t-s) f(w(s)) d s .
$$

defined for small positive $0<t<t_{1}$, with $x(t) \rightarrow x_{0}$ as $t \rightarrow 0+$. If $x_{0} \in D(A)$ and $f$ is continuously differentiable, then the solution is also a strict solution (i.e $x:\left(0, t_{1}\right) \rightarrow X$ is $C^{1}, x(t) \in D(A)$, for $0<t<t_{1}$ and the differential equation (1.2) is satisfied). If $A$ is bounded, the solution is defined in a open interval around 0 (see [9]).

Define, for $\lambda>0$,

$$
f_{\lambda}(\varphi)=\left\{\begin{array}{c}
f(\varphi), \text { if }\|\varphi\| \leq \lambda, \\
f\left(\frac{\lambda \varphi}{\|\varphi\|}\right), \text { if }\|\varphi\|>\lambda .
\end{array}\right.
$$

The properties on $f$ and $\eta$ ensure the existence of a non decreasing continuous function $\nu(\lambda), \lambda \geq 0, \nu(0)=0$ such that, for every $\varphi, \psi \in X$

$$
\begin{aligned}
\left\|f_{\lambda}(\varphi)\right\| & \leq \nu(\lambda) \lambda, \\
\left\|f_{\lambda}(\varphi)-f_{\lambda}(\psi)\right\| & \leq \nu(\lambda)\|\varphi-\psi\| .
\end{aligned}
$$

Thus, to study (1.3) locally it is enough to investigate the global behavior of the equation

$$
w(t)=T(t) w(0)+\int_{0}^{t} T(t-s) f_{\lambda}(w(s)) d s .
$$


This paper is organized as follows. In Section 2, we recall some results from [2]. In Section 3, we to prove the continuity of the invariant manifolds, with respect to a parameter.

\section{Existence of invariant manifolds}

We state below some results proved in [2], for completeness. We give below a slightly modified proof of the first result, just to introduce the ideas that will be used in the sequel.

Lemma 2.1. Let $\tau>0$ and $w:[0, \tau] \rightarrow X$ be continuous solutions of (1.3). Then

$$
y(t)=w(t+\tau), \forall t \in[-\tau, 0]
$$

satisfies

$$
T(-t) y(t)=y(0)+\int_{0}^{t} T(-s) f(y(s)) d s, \forall t \in[-\tau, 0] .
$$

Conversely, if (BU) holds and y satisfies (2.6), then $w$ satisfies (1.3) in $[0, \tau]$.

Proof Suppose that $w$ satisfies (1.3) and let $y$ be given by (2.5). Then, if $t \in[-\tau, 0]$, it follows that

$$
\begin{aligned}
T(-t) y(t) & =T(-t) w(t+\tau) \\
& =T(-t)\left[T(t+\tau) w(0)+\int_{0}^{t+\tau} T(t+\tau-s) f(w(s)) d s\right] \\
& =T(\tau) w(0)+\int_{0}^{t+\tau} T(\tau-s) f(w(s)) d s
\end{aligned}
$$

But, from (1.3) we have

$$
T(\tau) w(0)=w(\tau)-\int_{0}^{\tau} T(\tau-s) f(w(s)) d s .
$$

Therefore 


$$
\begin{aligned}
T(-t) y(t) & =w(\tau)-\int_{0}^{\tau} T(\tau-s) f(w(s)) d s+\int_{0}^{t+\tau} T(\tau-s) f(w(s)) d s \\
& =y(0)+\int_{\tau}^{t+\tau} T(\tau-s) f(w(s)) d s \\
& =y(0)+\int_{\tau}^{t+\tau} T(\tau-s) f(w(s)) d s \\
& =y(0)+\int_{0}^{t} T(-r) f(w(r+\tau)) d r \\
& =y(0)+\int_{0}^{t} T(-r) f(y(r)) d r
\end{aligned}
$$

that is, $y$ satisfies (2.6). Conversely, suppose that $y$ satisfies (2.6). Then

$$
y(0)=T(-t) y(t)-\int_{0}^{t} T(-s) f(y(s)) d s, t \in[-\tau, 0],
$$

which, with $t=-\tau$, becomes

$$
y(0)=T(\tau) y(-\tau)-\int_{0}^{-\tau} T(-s) f(y(s)) d s .
$$

Since $y(0)=w(\tau)$ and $y(-\tau)=w(0)$, it follows that

$$
w(\tau)=T(\tau) w(0)-\int_{0}^{-\tau} T(-s) f(w(s+\tau)) d s .
$$

On the other hand, from (2.6), we have

$$
T(-t) w(t+\tau)=w(\tau)+\int_{0}^{t} T(-s) f(w(s+\tau)) d s .
$$

Letting $-t=\tau-\theta$, we obtain

$$
T(\tau-\theta) w(\theta)=w(\tau)+\int_{0}^{\theta-\tau} T(-s) f(w(s+\tau)) d s .
$$

Using (2.7), we obtain

$$
\begin{aligned}
T(\tau-\theta) w(\theta) & =T(\tau) w(0)-\int_{0}^{-\tau} T(-s) f(w(s+\tau)) d s \\
& +\int_{0}^{\theta-\tau} T(-s) f(w(s+\tau)) d s \\
& =T(\tau) w(0)-\int_{\theta-\tau}^{-\tau} T(-s) f(w(s+\tau)) d s
\end{aligned}
$$


Thus

$$
T(\tau-\theta)\left[w(\theta)-T(\theta) w(0)+\int_{\theta-\tau}^{-\tau} T(\theta-\tau-s) f(w(s+\tau)) d s\right]=0 .
$$

Changing variables to $r=s+\tau$, we have

$$
T(\tau-\theta)\left[w(\theta)-T(\theta) w(0)-\int_{0}^{\theta} T(\theta-r) f(w(r)) d r\right]=0 .
$$

Thus, by (BU), it follows that

$$
w(\theta)-T(\theta) w(0)-\int_{0}^{\theta} T(\theta-r) f(w(r)) d r=0 .
$$

Therefore, $w$ satisfies (1.3).

Definition 2.2. A solution of (1.3) in an interval $[-\tau, 0], \tau>0$ is a function $y:[-\tau, 0] \rightarrow X$ such that $w$, given by (2.5), is a solution of (1.3) in $[0, \tau]$.

In the definition below, $B(\varphi, \varepsilon)$ denotes the ball of radius $\varepsilon$ and center at $\varphi$.

Definition 2.3. A subset $K \subset X$ is said to be locally positively invariant under the flow of (1.3) if there exists $\varepsilon>0$ such that, for any $\varphi \in K \cap$ $B(\varphi, \varepsilon)$ :

(i) for sufficiently small $t>0$ a solution $w(t)$, of (1.3), exists with $w(0)=$ $\varphi$;

(ii) if for $\tau>0, w(t)$ exists and belongs to $B(\varphi, \varepsilon)$ for all $t \in[0, \tau]$ then $w(t) \in K$, for all $t \in[0, \tau]$.

Negatively invariant subsets are defined by substituting $<$ for $>$ and $t \in[0, \tau]$ for $t \in[\tau, 0]$ in (i) and (ii).

Definition 2.4. Suppose that a Banach space $Y$ is decomposed as $Y=$ $\pi_{1} Y \oplus \pi_{2} Y$ for continuous linear projection operators $\pi_{1}$ and $\pi_{2}$. Then, a subset $S \subset Y$ of $Y$ containing $y_{0}$ is said to be tangent to $\pi_{2} Y$ at $y_{0}$ if

$$
\frac{\left\|\pi_{1}\left(y-y_{0}\right)\right\|}{\left\|\pi_{2}\left(y-y_{0}\right)\right\|} \rightarrow 0 \text {, as } y \rightarrow y_{0} \text { in } S \text {. }
$$

The following results has been proved in [2].

Theorem 2.5. Assume the hypotheses (H1), (H2), (H3) and (H4) hold. Let $\varepsilon>0$ be such that $\min \left(a_{-}, a_{+}\right)>\varepsilon$. Then, for $\delta>0$ sufficiently small, there exist locally invariant sets

$$
S=\left\{\varphi \in B(0, \delta):\left\|\varphi_{-}\right\|<\frac{\delta}{2 K}, \varphi_{0}+\varphi_{+}=p_{\lambda}\left(\varphi_{-}\right)\right\},
$$


and

$$
U=\left\{\varphi \in B(0, \delta):\left\|\varphi_{+}\right\|<\frac{\delta}{2 K}, \varphi_{-}+\varphi_{0}=q_{\lambda}\left(\varphi_{+}\right)\right\},
$$

termed the stable and unstable manifold respectively, where $p_{\lambda}, q_{\lambda}$ are Lipschitz function defined for $\left\|\varphi_{-}\right\|<\frac{\delta}{2 K},\left\|\varphi_{+}\right\|<\frac{\delta}{2 K}$, respectively. If $\varphi \in S$ then a unique solution $w(t)$ of (1.3) with $w(0)=\varphi$ exists for $t \geq 0$ and

$$
\|w(t)\| \leq 2 K e^{-\left(a_{-}-\varepsilon\right) t}\left\|w_{-}(0)\right\|, t \geq 0 .
$$

If the hypothesis (BU) holds and $\varphi \in U$ then an unique solution $w(t)$ of (1.3) with $w(0)=\varphi$ exists for $t \leq 0$ and

$$
\|w(t)\| \leq 2 K e^{\left(a_{+}-\varepsilon\right) t}\left\|w_{+}(0)\right\|, t \leq 0 .
$$

Furthermore, $S$ is tangent at zero to $\pi_{-} X, U$ is tangent at zero to $\pi_{+} X$ and $\left(p_{\lambda}, w_{+}^{p_{\lambda}}\right)$ is the unique solution of the system

$$
\begin{gathered}
w_{-}(t)=T(t) \varphi_{-}+\int_{0}^{t} T(t-s) \pi_{-} f_{\lambda}\left(w_{-}(s)+p_{\lambda}\left(w_{-}(s)\right)\right) d s, \\
p_{\lambda}\left(\varphi_{-}\right)=\int_{\infty}^{0} T(-s)\left(\pi_{0}+\pi_{+}\right) f_{\lambda}\left(w_{-}(s)+p_{\lambda}\left(w_{-}(s)\right)\right) d s, t \geq 0
\end{gathered}
$$

and $\left(q_{\lambda}, w_{+}^{q_{\lambda}}\right)$ is the unique solution of the system

$$
\begin{gathered}
q_{\lambda}\left(\varphi_{+}\right)=\int_{-\infty}^{0} T(-s)\left(\pi_{-}+\pi_{0}\right) f_{\lambda}\left(w_{+}(s)+q_{\lambda}\left(w_{+}(s)\right)\right) d s, \\
w_{+}(t)=T(t) \varphi_{+}+\int_{0}^{t} T(t-s) \pi_{+} f_{\lambda}\left(w_{+}(s)+q_{\lambda}\left(w_{+}(s)\right)\right) d s, t \leq 0,
\end{gathered}
$$

The functions $p_{\lambda}, q_{\lambda}$ have Lipschitz constants smaller or equal to $1, p_{\lambda}(0)=$ $0, q_{\lambda}(0)=0$ and $K$ is the constant given in the hypothesis (2.c).

In what follows, we denote by $B(0, \delta), B_{\left(\pi_{-} \oplus \pi_{0}\right)}(0, \delta), B_{\left(\pi_{0} \oplus \pi_{+}\right)}(0, \delta)$, the ball of radius $\delta$ and center in the origin of $X, \pi_{-} X \oplus \pi_{0} X, \pi_{0} X \oplus \pi_{+} X$, respectively.

Theorem 2.6. Assume the hypotheses (H1), (H2), (H3) and (H4) hold. Then, there exists $\delta>0$ and sets

$$
\begin{aligned}
& W^{* s}=\left\{\varphi \in X:\left\|\varphi_{-}+\varphi_{0}\right\|<\delta, \varphi_{+}=p^{*}\left(\varphi_{-}+\varphi_{0}\right)\right\}, \\
& W^{* u}=\left\{\varphi \in X:\left\|\varphi_{0}+\varphi_{+}\right\|<\delta, \varphi_{-}=q^{*}\left(\varphi_{0}+\varphi_{+}\right)\right\},
\end{aligned}
$$

termed the center-stable and center-unstable manifolds of (1.1), respectively, where $p^{*}$ and $q^{*}$ are Lipschitz functions defined on $B_{\left(\pi_{-} \oplus \pi_{0}\right)}(0, \delta) \subset \pi_{-} X \oplus \pi_{0} X, B_{\left(\pi_{0} \oplus \pi_{+}\right)}(0, \delta) \subset \pi_{0} X \oplus \pi_{+} X$, respectively; The set $W^{* s}$ is locally positively invariant under the flow (1.3), while if (BU) holds $W^{* u}$ is locally negatively invariant. Any solution of (1.3) which 
exists and remains in $B(0, \delta)$ fort $\geq 0$ lies on $W^{* s}$, and any solution of (1.3) which exists and remains in $B(0, \delta)$ for $t \leq 0$ lies on $W^{* u}$. Furthermore, the tangent space to $W^{* s}$ at zero is $\pi_{-} X \oplus \pi_{0} X$ and the tangent space to $W^{* u}$ at zero is $\pi_{0} X \oplus \pi_{+} X$.

Proof This theorem has also been proved in [2], where $W^{* s}$ for (1.4) is shown to exist, for $\lambda$ small enough to imply

$$
\max \left\{\frac{2 K^{2} \nu(\lambda)}{a_{+}-a_{0}-4 K \nu(\lambda)}, \frac{2(K \nu(\lambda))^{2}}{a_{0}\left(a_{+}-a_{0}-4 K \nu(\lambda)\right)}+\frac{K \nu(\lambda)}{a_{+}}\right\}<1,
$$

and $p_{\lambda}^{*}$, for $t \geq 0$, is the unique solution of the system below

$$
\begin{gathered}
w_{-}(t)=T(t) \varphi_{-}+\int_{0}^{t} T(t-s) \pi_{-} f_{\lambda}\left(w_{-}(s)+w_{0}(s)+p_{\lambda}^{*}\left(w_{-}(s)+w_{0}(s)\right)\right) d s, \\
w_{0}(t)=T(t) \varphi_{0}+\int_{0}^{t} T(t-s) \pi_{0} f_{\lambda}\left(w_{-}(s)+w_{0}(s)+p_{\lambda}^{*}\left(w_{-}(s)+w_{0}(s)\right)\right) d s, \\
p_{\lambda}^{*}\left(\varphi_{-}+\varphi_{0}\right)=\int_{\infty}^{0} T(-s) \pi_{+} f_{\lambda}\left(w_{-}(s)+w_{0}(s)+p_{\lambda}^{*}\left(w_{-}(s)+w_{0}(s)\right)\right) d s,
\end{gathered}
$$

where $w_{-}(t)=\pi_{-} w(t), w_{0}(t)=\pi_{0} w(t)$ e $w(t)$ satisfies (1.4). Furthermore, $p_{\lambda}^{*}$ has Lipschitz constant smaller or equal to 1 and $p_{\lambda}^{*}(0)=0$.

Similarly, $W^{* u}$ can be shown to exist as a graph of $q_{\lambda}^{*}$, the unique solution of the system

$$
\begin{gathered}
q_{\lambda}^{*}\left(\varphi_{0}+\varphi_{+}\right)=\int_{-\infty}^{0} T(-s) \pi_{-} f_{\lambda}\left(q_{\lambda}^{*}\left(w_{0}(s)+w_{+}(s)\right)+w_{0}(s)+w_{+}(s)\right) d s, \\
w_{0}(t)=T(t) \varphi_{0}+\int_{0}^{t} T(t-s) \pi_{0} f_{\lambda}\left(q_{\lambda}^{*}\left(w_{0}(s)+w_{+}(s)\right)+w_{0}(s)+w_{+}(s)\right) d s, \\
w_{+}(t)=T(t) \varphi_{+}+\int_{0}^{t} T(t-s) \pi_{+} f_{\lambda}\left(q_{\lambda}^{*}\left(w_{0}(s)+w_{+}(s)\right)+w_{0}(s)+w_{+}(s)\right) d s,
\end{gathered}
$$

for $t \leq 0$, where $w_{0}(t)=\pi_{0} w(t), w_{+}(t)=\pi_{+} w(t)$ and $w(t)$ satisfies (1.4).

\section{Continuity of the invariant manifolds}

We prove in this section that the manifolds of the previous section are continuous with respect to the parameter $\varepsilon$.

3.1. Continuity of the stable (unstable) manifolds. We now state one of the main results of this section. 
Theorem 3.1. (Continuity of the stable (unstable) manifolds). Suppose that the functions $f_{\lambda}$, in (1.4), also depend on a parameter $\varepsilon \in \Lambda$, where $\Lambda$ is an open set in a Banach space, and $f_{\lambda}=f_{\lambda}^{\varepsilon}$ satisfies the estimates

$$
\begin{gathered}
\left\|f_{\lambda}^{\varepsilon}(u)-f_{\lambda}^{\varepsilon_{0}}(u)\right\| \leq C_{1}(\varepsilon)\|u\|, C_{1}(\varepsilon) \rightarrow 0, \varepsilon \rightarrow \varepsilon_{0} \\
\left\|f_{\lambda}^{\varepsilon}(u)-f_{\lambda}^{\varepsilon}(v)\right\| \leq \nu(\lambda)\|u-v\|, \text { for each } \varepsilon \in \Lambda,
\end{gathered}
$$

where $\nu(\cdot)$ is a non decreasing continuous function with $\nu(0)=0$. Then the stable (unstable) manifold $S^{\varepsilon},\left(U^{\varepsilon}\right)$, is continuous with respect to the parameter $\varepsilon$ at $\varepsilon_{0}$.

Proof We prove the continuity of $S^{\varepsilon}$; the proof for $U^{\varepsilon}$ is analogous. By Theorem 2.5, $S^{\varepsilon}$ is the graph of a Lipschitz function $p_{\lambda}=p_{\lambda}^{\varepsilon}$, where $\left(p_{\lambda}, w_{-}^{p_{\lambda}}\right)$ is the unique solution of (2.8). From (2.8) and (3.10), we have

$$
\begin{aligned}
& \left\|w_{-}(t, \varepsilon)\right\| \\
& \leq K e^{-a_{-} t}\left\|\varphi_{-}\right\|+\int_{0}^{t} K e^{-a_{-}(t-s)} \nu(\lambda)\left\|w_{-}(s, \varepsilon)+p_{\lambda}^{\varepsilon}\left(w_{-}(s, \varepsilon)\right)\right\| d s \\
& \leq K e^{-a_{-} t}\left\|\varphi_{-}\right\|+\int_{0}^{t} K e^{-a_{-}(t-s)} \nu(\lambda)\left[\left\|w_{-}(s, \varepsilon)\right\|+\left\|p_{\lambda}^{\varepsilon}\left(w_{-}(s, \varepsilon)\right)\right\|\right] d s \\
& \leq K e^{-a_{-} t}\left\|\varphi_{-}\right\|+\int_{0}^{t} 2 K \nu(\lambda) e^{-a_{-}(t-s)}\left\|w_{-}(s, \varepsilon)\right\| d s .
\end{aligned}
$$

By Gronwall's Lemma, we obtain

$$
\left\|w_{-}(t, \varepsilon)\right\| \leq K\left\|\varphi_{-}\right\| e^{-\left(a_{-}-2 K \nu(\lambda)\right) t}, t \geq 0
$$

We will use the metric $\rho$ given by

$$
\rho\left(h_{1}, h_{2}\right)=\sup _{\substack{\varphi \in X \\ \varphi_{-} \neq 0}} \frac{\left\|h_{1}\left(\varphi_{-}\right)-h_{2}\left(\varphi_{-}\right)\right\|}{\left\|\varphi_{-}\right\|},
$$

equipped with which, the set

$$
G=\left\{h: \pi_{-} X \rightarrow \pi_{0} X \oplus \pi_{+} X,\left\|h\left(\varphi_{-}\right)-h\left(\psi_{+}\right)\right\| \leq\left\|\varphi_{-}-\psi_{+}\right\|,\right.
$$

$\forall \varphi, \psi \in X, h(0)=0\}$ becomes a complete metric space. 
Let $\theta(t)=\left\|w_{-}(t, \varepsilon)-w_{-}\left(t, \varepsilon_{0}\right)\right\|, t \geq 0$. Then

$$
\begin{aligned}
\theta(t) \leq \int_{0}^{t} \| T(t-s) \pi_{-}\left\{f_{\lambda}^{\varepsilon}\left[w_{-}(s, \varepsilon)+p_{\lambda}^{\varepsilon}\left(w_{-}(s, \varepsilon)\right)\right]\right. \\
\left.\quad-f_{\lambda}^{\varepsilon_{0}}\left[w_{-}\left(s, \varepsilon_{0}\right)+p_{\lambda}^{\varepsilon_{0}}\left(w_{-}\left(s, \varepsilon_{0}\right)\right)\right]\right\} \| d s \\
\leq \int_{0}^{t} K e^{-(t-s) a_{-}} \| f_{\lambda}^{\varepsilon}\left[w_{-}(s, \varepsilon)+p_{\lambda}^{\varepsilon}\left(w_{-}(s, \varepsilon)\right)\right] \\
\quad-f_{\lambda}^{\varepsilon_{0}}\left[w_{-}\left(s, \varepsilon_{0}\right)+p_{\lambda}^{\varepsilon_{0}}\left(w_{-}\left(s, \varepsilon_{0}\right)\right)\right] \| d s \\
\leq \int_{0}^{t} K e^{-(t-s) a_{-}} \| f_{\lambda}^{\varepsilon}\left[w_{-}(s, \varepsilon)+p_{\lambda}^{\varepsilon}\left(w_{-}(s, \varepsilon)\right)\right] \\
\quad-f_{\lambda}^{\varepsilon}\left[w_{-}\left(s, \varepsilon_{0}\right)+p_{\lambda}^{\varepsilon_{0}}\left(w_{-}\left(s, \varepsilon_{0}\right)\right)\right] \| d s \\
+\int_{0}^{t} K e^{-(t-s) a_{-}} \| f_{\lambda}^{\varepsilon}\left[w_{-}\left(s, \varepsilon_{0}\right)+p_{\lambda}^{\varepsilon_{0}}\left(w_{-}\left(s, \varepsilon_{0}\right)\right)\right] \\
-f_{\lambda}^{\varepsilon_{0}}\left[w_{-}\left(s, \varepsilon_{0}\right)+p_{\lambda}^{\varepsilon_{0}}\left(w_{-}\left(s, \varepsilon_{0}\right)\right)\right] \| d s .
\end{aligned}
$$

From (3.9) and (3.10), it follows that

$$
\begin{gathered}
\theta(t) \leq \int_{0}^{t} K \nu(\lambda) e^{-(t-s) a_{-}}\left[\left\|w_{-}(s, \varepsilon)-w_{-}\left(s, \varepsilon_{0}\right)\right\|+\right. \\
\left.\left\|p_{\lambda}^{\varepsilon}\left(w_{-}(s, \varepsilon)\right)-p_{\lambda}^{\varepsilon_{0}}\left(w_{-}\left(s, \varepsilon_{0}\right)\right)\right\|\right] d s \\
\quad+\int_{0}^{t} K e^{-(t-s) a_{-}} C_{1}(\varepsilon)\left\|w_{-}\left(s, \varepsilon_{0}\right)+p_{\lambda}^{\varepsilon_{0}}\left(w_{-}\left(s, \varepsilon_{0}\right)\right)\right\| d s \\
=\int_{0}^{t} K \nu(\lambda) e^{-(t-s) a_{-}}\left[\theta(s)+\left\|p_{\lambda}^{\varepsilon}\left(w_{-}(s, \varepsilon)\right)-p_{\lambda}^{\varepsilon_{0}}\left(w_{-}\left(s, \varepsilon_{0}\right)\right)\right\|\right] d s \\
\quad+\int_{0}^{t} K e^{-(t-s) a_{-}} C_{1}(\varepsilon)\left\|w_{-}\left(s, \varepsilon_{0}\right)+p_{\lambda}^{\varepsilon_{0}}\left(w_{-}\left(s, \varepsilon_{0}\right)\right)\right\| d s .
\end{gathered}
$$

Using that $p_{\lambda}^{\varepsilon_{0}}$ is Lipschitz with Lipschitz constant $\leq 1$ and $p_{\lambda}^{\varepsilon_{0}}(0)=0$, we obtain

$$
\begin{aligned}
\theta(t) & \leq \int_{0}^{t} K \nu(\lambda) e^{-(t-s) a_{-}}\left[\theta(s)+\left\|p_{\lambda}^{\varepsilon}\left(w_{-}(s, \varepsilon)\right)-p_{\lambda}^{\varepsilon_{0}}\left(w_{-}\left(s, \varepsilon_{0}\right)\right)\right\|\right] d s \\
& +\int_{0}^{t} 2 K e^{-(t-s) a_{-}} C_{1}(\varepsilon)\left\|w_{-}\left(s, \varepsilon_{0}\right)\right\| d s .
\end{aligned}
$$


Now, using the same argument for $p_{\lambda}^{\varepsilon}$, we have

$$
\begin{aligned}
\| p_{\lambda}^{\varepsilon}\left(w_{-}(s, \varepsilon)\right)- & p_{\lambda}^{\varepsilon_{0}}\left(w_{-}\left(s, \varepsilon_{0}\right)\right) \| \\
\leq & \left\|p_{\lambda}^{\varepsilon}\left(w_{-}(s, \varepsilon)\right)-p_{\lambda}^{\varepsilon}\left(w_{-}\left(s, \varepsilon_{0}\right)\right)\right\| \\
& +\left\|p_{\lambda}^{\varepsilon}\left(w_{-}\left(s, \varepsilon_{0}\right)\right)-p_{\lambda}^{\varepsilon_{0}}\left(w_{-}\left(s, \varepsilon_{0}\right)\right)\right\| \\
\leq & \left\|w_{-}(s, \varepsilon)-w_{-}\left(s, \varepsilon_{0}\right)\right\| \\
& +\left\|p_{\lambda}^{\varepsilon}\left(w_{-}\left(s, \varepsilon_{0}\right)\right)-p_{\lambda}^{\varepsilon_{0}}\left(w_{-}\left(s, \varepsilon_{0}\right)\right)\right\| \\
& =\theta(s)+\left\|p_{\lambda}^{\varepsilon}\left(w_{-}\left(s, \varepsilon_{0}\right)\right)-p_{\lambda}^{\varepsilon_{0}}\left(w_{-}\left(s, \varepsilon_{0}\right)\right)\right\| \\
& =\theta(s)+\left\|w_{-}\left(s, \varepsilon_{0}\right)\right\| \frac{\left\|p_{\lambda}^{\varepsilon}\left(w_{-}\left(s, \varepsilon_{0}\right)\right)-p_{\lambda}^{\varepsilon_{0}}\left(w_{-}\left(s, \varepsilon_{0}\right)\right)\right\|}{\left\|w_{-}\left(s, \varepsilon_{0}\right)\right\|} \\
& \leq \theta(s)+\left\|w_{-}\left(s, \varepsilon_{0}\right)\right\| \rho\left(p_{\lambda}^{\varepsilon}, p_{\lambda}^{\varepsilon_{0}}\right) .
\end{aligned}
$$

Therefore

$$
\begin{aligned}
\theta(t) & \leq \int_{0}^{t} K \nu(\lambda) e^{-(t-s) a_{-}}\left[\theta(s)+\theta(s)+\left\|w_{-}\left(s, \varepsilon_{0}\right)\right\| \rho\left(p_{\lambda}^{\varepsilon}, p_{\lambda}^{\varepsilon_{0}}\right)\right] d s \\
& +\int_{0}^{t} 2 K C_{1}(\varepsilon) e^{-(t-s) a_{-}}\left\|w_{-}\left(s, \varepsilon_{0}\right)\right\| d s \\
& =\int_{0}^{t} 2 K \nu(\lambda) e^{-(t-s) a_{-}} \theta(s) d s \\
& +\int_{0}^{t} K \nu(\lambda) e^{-(t-s) a_{-}}\left\|w_{-}\left(s, \varepsilon_{0}\right)\right\| \rho\left(p_{\lambda}^{\varepsilon}, p_{\lambda}^{\varepsilon_{0}}\right) d s \\
& +\int_{0}^{t} 2 K C_{1}(\varepsilon) e^{-(t-s) a_{-}}\left\|w_{-}\left(s, \varepsilon_{0}\right)\right\| d s .
\end{aligned}
$$


Using (3.11), we obtain

$$
\begin{aligned}
\theta(t) & \leq \int_{0}^{t} 2 K \nu(\lambda) e^{-(t-s) a_{-}} \theta(s) d s \\
& +\int_{0}^{t} K \nu(\lambda) e^{-(t-s) a_{-}} \rho\left(p_{\lambda}^{\varepsilon}, p_{\lambda}^{\varepsilon_{0}}\right) K\left\|\varphi_{-}\right\| e^{-\left(a_{-}-2 K \nu(\lambda)\right) s} d s \\
& +\int_{0}^{t} 2 K C_{1}(\varepsilon) e^{-(t-s) a_{-}} K\left\|\varphi_{-}\right\| e^{-\left(a_{-}-2 K \nu(\lambda)\right) s} d s \\
& =\int_{0}^{t} 2 K \nu(\lambda) e^{-(t-s) a_{-}} \theta(s) d s \\
& +K^{2}\left\|\varphi_{-}\right\| \nu(\lambda) \rho\left(p_{\lambda}^{\varepsilon}, p_{\lambda}^{\varepsilon_{0}}\right) e^{-a_{-} t} \int_{0}^{t} e^{2 K \nu(\lambda) s} d s \\
& +2 K^{2}\left\|\varphi_{-}\right\| C_{1}(\varepsilon) e^{-a_{-} t} \int_{0}^{t} e^{2 K \nu(\lambda) s} d s .
\end{aligned}
$$

Thus

$$
\begin{aligned}
e^{a_{-} t} \theta(t) & \leq \int_{0}^{t} 2 K \nu(\lambda) e^{a_{-} s} \theta(s) d s+K^{2}\left\|\varphi_{-}\right\| \nu(\lambda) \rho\left(p_{\lambda}^{\varepsilon}, p_{\lambda}^{\varepsilon_{0}}\right) \int_{0}^{t} e^{2 K \nu(\lambda) s} d s \\
& +2 K^{2}\left\|\varphi_{-}\right\| C_{1}(\varepsilon) \int_{0}^{t} e^{2 K \nu(\lambda) s} d s \\
& \leq \int_{0}^{t} 2 K \nu(\lambda) e^{a_{-} s} \theta(s) d s+\frac{K\left\|\varphi_{-}\right\| \nu(\lambda)}{2 \nu(\lambda)} \rho\left(p_{\lambda}^{\varepsilon}, p_{\lambda}^{\varepsilon_{0}}\right) e^{2 K \nu(\lambda) t} \\
& +\frac{K\left\|\varphi_{-}\right\|}{\nu(\lambda)} C_{1}(\varepsilon) e^{2 K \nu(\lambda) t} .
\end{aligned}
$$

From the generalized Gronwall's Lemma, (see [12]), it follows that

$$
e^{a_{-} t} \theta(t) \leq e^{2 K \nu(\lambda) t}\left[\frac{K\left\|\varphi_{-}\right\|}{2} \rho\left(p_{\lambda}^{\varepsilon}, p_{\lambda}^{\varepsilon_{0}}\right) e^{2 K \nu(\lambda) t}+\frac{K\left\|\varphi_{-}\right\| C_{1}(\varepsilon)}{\nu(\lambda)} e^{2 K \nu(\lambda) t}\right] .
$$

\section{Hence}

$$
\theta(t) \leq \frac{K\left\|\varphi_{-}\right\|}{2} \rho\left(p_{\lambda}^{\varepsilon}, p_{\lambda}^{\varepsilon_{0}}\right) e^{-\left(a_{-}-4 K \nu(\lambda)\right) t}+\frac{K\left\|\varphi_{-}\right\| C_{1}(\varepsilon)}{\nu(\lambda)} e^{-\left(a_{-}-4 K \nu(\lambda)\right) t}
$$


Now

$$
\begin{aligned}
\left\|p_{\lambda}^{\varepsilon}\left(\varphi_{-}\right)-p_{\lambda}^{\varepsilon_{0}}\left(\varphi_{-}\right)\right\| & \leq \int_{0}^{\infty} \| T(-s) \pi_{0}\left[f_{\lambda}^{\varepsilon}\left(w_{-}(s, \varepsilon)+p_{\lambda}^{\varepsilon}\left(w_{-}(s, \varepsilon)\right)\right)\right. \\
& \left.-f_{\lambda}^{\varepsilon_{0}}\left(w_{-}\left(s, \varepsilon_{0}\right)+p_{\lambda}^{\varepsilon_{0}}\left(w_{-}\left(s, \varepsilon_{0}\right)\right)\right)\right] \| d s \\
& +\int_{0}^{\infty} \| T(-s) \pi_{+}\left[f_{\lambda}^{\varepsilon}\left(w_{-}(s, \varepsilon)+p_{\lambda}^{\varepsilon}\left(w_{-}(s, \varepsilon)\right)\right)\right. \\
& \left.-f_{\lambda}^{\varepsilon_{0}}\left(w_{-}\left(s, \varepsilon_{0}\right)+p_{\lambda}^{\varepsilon_{0}}\left(w_{-}\left(s, \varepsilon_{0}\right)\right)\right)\right] \| d s \\
& \leq \int_{0}^{\infty} K e^{a_{0} s} \| f_{\lambda}^{\varepsilon}\left[w_{-}(s, \varepsilon)+p_{\lambda}^{\varepsilon}\left(w_{-}(s, \varepsilon)\right)\right] \\
& -f_{\lambda}^{\varepsilon_{0}}\left[w_{-}\left(s, \varepsilon_{0}\right)+p_{\lambda}^{\varepsilon_{0}}\left(w_{-}\left(s, \varepsilon_{0}\right)\right)\right] \| d s \\
& +\int_{0}^{\infty} K e^{-a_{+} s} \| f_{\lambda}^{\varepsilon}\left[w_{-}(s, \varepsilon)+p_{\lambda}^{\varepsilon}\left(w_{-}(s, \varepsilon)\right)\right] \\
& -f_{\lambda}^{\varepsilon_{0}}\left[w_{-}\left(s, \varepsilon_{0}\right)+p_{\lambda}^{\varepsilon_{0}}\left(w_{-}\left(s, \varepsilon_{0}\right)\right)\right] \| d s .
\end{aligned}
$$

Using that, for $s>0,-a_{+} s<a_{0} s$, it follows that

$$
\begin{aligned}
\left\|p_{\lambda}^{\varepsilon}\left(\varphi_{-}\right)-p_{\lambda}^{\varepsilon_{0}}\left(\varphi_{+}\right)\right\| & \leq \int_{0}^{\infty} 2 K e^{a_{0} s} \| f_{\lambda}^{\varepsilon}\left[w_{-}(s, \varepsilon)+p_{\lambda}^{\varepsilon}\left(w_{-}(s, \varepsilon)\right)\right] \\
& -f_{\lambda}^{\varepsilon_{0}}\left[w_{-}\left(s, \varepsilon_{0}\right)+p_{\lambda}^{\varepsilon_{0}}\left(w_{-}\left(s, \varepsilon_{0}\right)\right)\right] \| d s
\end{aligned}
$$

Subtracting and summing the term

$$
f_{\lambda}^{\varepsilon}\left[w_{-}\left(s, \varepsilon_{0}\right)+p_{\lambda}^{\varepsilon_{0}}\left(w_{-}\left(s, \varepsilon_{0}\right)\right)\right]
$$

we obtain

$$
\begin{aligned}
\left\|p_{\lambda}^{\varepsilon}\left(\varphi_{-}\right)-p_{\lambda}^{\varepsilon_{0}}\left(\varphi_{+}\right)\right\| & \leq \int_{0}^{\infty} 2 K e^{a_{0} s} \| f_{\lambda}^{\varepsilon}\left[w_{-}(s, \varepsilon)+p_{\lambda}^{\varepsilon}\left(w_{-}(s, \varepsilon)\right)\right] \\
& -f_{\lambda}^{\varepsilon}\left[w_{-}\left(s, \varepsilon_{0}\right)+p_{\lambda}^{\varepsilon_{0}}\left(w_{-}\left(s, \varepsilon_{0}\right)\right)\right] \| d s \\
& +\int_{0}^{\infty} 2 K e^{a_{0} s} \| f_{\lambda}^{\varepsilon}\left[w_{-}\left(s, \varepsilon_{0}\right)+p_{\lambda}^{\varepsilon_{0}}\left(w_{-}\left(s, \varepsilon_{0}\right)\right)\right] \\
& -f_{\lambda}^{\varepsilon_{0}}\left[w_{-}\left(s, \varepsilon_{0}\right)+p_{\lambda}^{\varepsilon_{0}}\left(w_{-}\left(s, \varepsilon_{0}\right)\right)\right] \| d s
\end{aligned}
$$


Using (3.9) and (3.10), it follows that

$$
\begin{aligned}
& \left\|p_{\lambda}^{\varepsilon}\left(\varphi_{-}\right)-p_{\lambda}^{\varepsilon_{0}}\left(\varphi_{-}\right)\right\| \\
& \leq \int_{0}^{\infty} 2 K \nu(\lambda) e^{a_{0} s}\left\{\left\|w_{-}(s, \varepsilon)-w_{-}\left(s, \varepsilon_{0}\right)\right\|\right. \\
& \left.\quad+\left\|p_{\lambda}^{\varepsilon}\left(w_{-}(s, \varepsilon)\right)-p_{\lambda}^{\varepsilon_{0}}\left(w_{-}\left(s, \varepsilon_{0}\right)\right)\right\|\right\} d s \\
& \quad+\int_{0}^{\infty} 2 K e^{a_{0} s} C_{1}(\varepsilon)\left\|w_{-}\left(s, \varepsilon_{0}\right)+p_{\lambda}^{\varepsilon_{0}}\left(w_{-}\left(s, \varepsilon_{0}\right)\right)\right\| d s \\
& =\int_{0}^{\infty} 2 K \nu(\lambda) e^{a_{0} s}\left\{\theta(s)+\left\|p_{\lambda}^{\varepsilon}\left(w_{-}(s, \varepsilon)\right)-p_{\lambda}^{\varepsilon_{0}}\left(w_{-}\left(s, \varepsilon_{0}\right)\right)\right\|\right\} d s \\
& \left.\left.\quad+\int_{0}^{\infty} 2 K e^{a_{0} s} C_{1}(\varepsilon)\left\|w_{-}\left(s, \varepsilon_{0}\right)+p_{\lambda}^{\varepsilon_{0}}\left(w_{-}\left(s, \varepsilon_{0}\right)\right)\right\| d s\right)\right\} d s \\
& \leq \int_{0}^{\infty} 2 K \nu(\lambda) e^{a_{0} s}\left\{\theta(s)+\left\|p_{\lambda}^{\varepsilon}\left(w_{-}(s, \varepsilon)\right)-p_{\lambda}^{\varepsilon_{0}}\left(w_{-}\left(s, \varepsilon_{0}\right)\right)\right\|\right\} d s \\
& \quad+\int_{0}^{\infty} 4 K e^{a_{0} s} C_{1}(\varepsilon)\left\|w_{-}\left(s, \varepsilon_{0}\right)\right\| d s .
\end{aligned}
$$

Using once again that

$$
\left\|p_{\lambda}^{\varepsilon}\left(w_{-}(s, \varepsilon)\right)-p_{\lambda}^{\varepsilon_{0}}\left(w_{-}\left(s, \varepsilon_{0}\right)\right)\right\| \leq \theta(s)+\left\|w_{-}\left(s, \varepsilon_{0}\right)\right\| \rho\left(p_{\lambda}^{\varepsilon}, p_{\lambda}^{\varepsilon_{0}}\right),
$$

we obtain

$$
\begin{aligned}
\left\|p_{\lambda}^{\varepsilon}\left(\varphi_{-}\right)-p_{\lambda}^{\varepsilon_{0}}\left(\varphi_{-}\right)\right\| & \leq \int_{0}^{\infty} 2 K \nu(\lambda) e^{a_{0} s}\left[2 \theta(s)+\left\|w_{-}\left(s, \varepsilon_{0}\right)\right\| \rho\left(p_{\lambda}^{\varepsilon}, p_{\lambda}^{\varepsilon_{0}}\right)\right] d s \\
& +\int_{0}^{\infty} 4 K C_{1}(\varepsilon) e^{a_{0} s}\left\|w_{-}\left(s, \varepsilon_{0}\right)\right\| d s .
\end{aligned}
$$

Now, using (3.11), it follows that

$$
\begin{aligned}
\left\|p_{\lambda}^{\varepsilon}\left(\varphi_{-}\right)-p_{\lambda}^{\varepsilon_{0}}\left(\varphi_{-}\right)\right\| & \leq \int_{0}^{\infty} 4 K \nu(\lambda) e^{a_{0} s} \theta(s) d s \\
& +\int_{0}^{\infty} 2 K^{2} \nu(\lambda)\left\|\varphi_{-}\right\| \rho\left(p_{\lambda}^{\varepsilon}, p_{\lambda}^{\varepsilon_{0}}\right) e^{-\left(a_{-}-a_{0}-2 K \nu(\lambda)\right) s} d s \\
& +\int_{0}^{\infty} 4 K^{2}\left\|\varphi_{-}\right\| C_{1}(\varepsilon) e^{-\left(a_{-}-a_{0}-2 K \nu(\lambda)\right) s} d s
\end{aligned}
$$

Thus,

$$
\left\|p_{\lambda}^{\varepsilon}\left(\varphi_{-}\right)-p_{\lambda}^{\varepsilon_{0}}\left(\varphi_{-}\right)\right\| \leq I_{1}+I_{2}+I_{3}
$$


where

$$
\begin{gathered}
I_{1}=\int_{0}^{\infty} 4 K \nu(\lambda) e^{a_{0} s} \theta(s) d s \\
I_{2}=\int_{0}^{\infty} 2 K^{2} \nu(\lambda)\left\|\varphi_{-}\right\| \rho\left(p_{\lambda}^{\varepsilon}, p_{\lambda}^{\varepsilon_{0}}\right) e^{-\left(a_{-}-a_{0}-2 K \nu(\lambda)\right) s} d s
\end{gathered}
$$

and

$$
I_{3}=\int_{0}^{\infty} 4 K^{2}\left\|\varphi_{-}\right\| C_{1}(\varepsilon) e^{-\left(a_{-}-a_{0}-2 K \nu(\lambda)\right) s} d s .
$$

Using the estimate obtained for $\theta(t)$ in (3.12), we obtain

$$
\begin{aligned}
I_{1} \leq \int_{0}^{\infty} 4 K \nu(\lambda) e^{a_{0} s}\left[\frac{K}{2}\left\|\varphi_{-}\right\| \rho\left(p_{\lambda}^{\varepsilon}, p_{\lambda}^{\varepsilon_{0}}\right) e^{-\left(a_{-}-4 K \nu(\lambda)\right) s}\right. \\
\left.\quad+\frac{K\left\|\varphi_{-}\right\|}{\nu(\lambda)} C_{1}(\varepsilon) e^{-\left(a_{-}-4 K \nu(\lambda)\right) s}\right] d s \\
=\int_{0}^{\infty} 2 K^{2}\left\|\varphi_{-}\right\| \nu(\lambda) \rho\left(p_{\lambda}^{\varepsilon}, p_{\lambda}^{\varepsilon_{0}}\right) e^{-\left(a_{-}-a_{0}-4 K \nu(\lambda)\right) s} d s \\
\quad+\int_{0}^{\infty} 4 K^{2}\left\|\varphi_{-}\right\| C_{1}(\varepsilon) e^{-\left(a_{-}-a_{0}-4 K \nu(\lambda)\right) s} d s \\
=\frac{2 K^{2} \nu(\lambda)\left\|\varphi_{-}\right\|}{a_{-}-a_{0}-4 K \nu(\lambda)} \rho\left(p_{\lambda}^{\varepsilon}, p_{\lambda}^{\varepsilon_{0}}\right)+\frac{4 K^{2}\left\|\varphi_{-}\right\|}{a_{-}-a_{0}-4 K \nu(\lambda)} C_{1}(\varepsilon) .
\end{aligned}
$$

Furthermore,

$$
\begin{aligned}
I_{2} & =\int_{0}^{\infty} 2 K^{2} \nu(\lambda)\left\|\varphi_{-}\right\| \rho\left(p_{\lambda}^{\varepsilon}, p_{\lambda}^{\varepsilon_{0}}\right) e^{-\left(a_{-}-a_{0}-2 K \nu(\lambda)\right) s} d s \\
& =\frac{2 K^{2} \nu(\lambda)\left\|\varphi_{-}\right\|}{a_{-}-a_{0}-2 K \nu(\lambda)} \rho\left(p_{\lambda}^{\varepsilon}, p_{\lambda}^{\varepsilon_{0}}\right)
\end{aligned}
$$

and

$$
\begin{aligned}
I_{3} & =\int_{0}^{\infty} 4 K^{2}\left\|\varphi_{-}\right\| C_{1}(\varepsilon) e^{-\left(a_{-}-a_{0}-2 K \nu(\lambda)\right) s} d s \\
& =\frac{4 K^{2}\left\|\varphi_{-}\right\|}{a_{-}-a_{0}-2 K \nu(\lambda)} C_{1}(\varepsilon) .
\end{aligned}
$$


Therefore

$$
\begin{aligned}
& \left\|p_{\lambda}^{\varepsilon}\left(\varphi_{-}\right)-p_{\lambda}^{\varepsilon_{0}}\left(\varphi_{-}\right)\right\| \\
& \leq \frac{2 K^{2} \nu(\lambda)\left\|\varphi_{-}\right\|}{a_{-}-a_{0}-4 K \nu(\lambda)} \rho\left(p_{\lambda}^{\varepsilon}, p_{\lambda}^{\varepsilon_{0}}\right)+\frac{4 K^{2}\left\|\varphi_{-}\right\|}{a_{-}-a_{0}-4 K \nu(\lambda)} C_{1}(\varepsilon) \\
& +\frac{2 K^{2} \nu(\lambda)\left\|\varphi_{-}\right\|}{a_{-}-a_{0}-2 K \nu(\lambda)} \rho\left(p_{\lambda}^{\varepsilon}, p_{\lambda}^{\varepsilon_{0}}\right)+\frac{4 K^{2}\left\|\varphi_{-}\right\|}{a_{-}-a_{0}-2 K \nu(\lambda)} C_{1}(\varepsilon) \\
& =\left[\frac{2 K^{2} \nu(\lambda)\left\|\varphi_{-}\right\|}{a_{-}-a_{0}-4 K \nu(\lambda)}+\frac{2 K^{2} \nu(\lambda)\left\|\varphi_{-}\right\|}{a_{-}-a_{0}-2 K \nu(\lambda)}\right] \rho\left(p_{\lambda}^{\varepsilon}, p_{\lambda}^{\varepsilon_{0}}\right) \\
& +\left[\frac{4 K^{2}\left\|\varphi_{-}\right\|}{a_{-}-a_{0}-4 K \nu(\lambda)}+\frac{4 K^{2}\left\|\varphi_{-}\right\|}{a_{-}-a_{0}-2 K \nu(\lambda)}\right] C_{1}(\varepsilon) .
\end{aligned}
$$

Hence

$$
\begin{aligned}
& \frac{\left\|p_{\lambda}^{\varepsilon}\left(\varphi_{-}\right)-p_{\lambda}^{\varepsilon_{0}}\left(\varphi_{-}\right)\right\|}{\left\|\varphi_{-}\right\|} \\
& \quad \leq\left[\frac{2 K^{2} \nu(\lambda)}{a_{-}-a_{0}-4 K \nu(\lambda)}+\frac{2 K^{2} \nu(\lambda)}{a_{-}-a_{0}-2 K \nu(\lambda)}\right] \rho\left(p_{\lambda}^{\varepsilon}, p_{\lambda}^{\varepsilon_{0}}\right) \\
& \quad+\left[\frac{4 K^{2}}{a_{-}-a_{0}-4 K \nu(\lambda)}+\frac{4 K^{2}}{a_{-}-a_{0}-2 K \nu(\lambda)}\right] C_{1}(\varepsilon),
\end{aligned}
$$

which implies

$$
\begin{aligned}
& \sup _{\substack{\varphi \in X \\
\varphi \neq \neq 0}} \frac{\left\|p_{\lambda}^{\varepsilon}\left(\varphi_{-}\right)-p_{\lambda}^{\varepsilon_{0}}\left(\varphi_{-}\right)\right\|}{\left\|\varphi_{-}\right\|} \\
& \leq\left[\frac{2 K^{2} \nu(\lambda)}{a_{-}-a_{0}-4 K \nu(\lambda)}+\frac{2 K^{2} \nu(\lambda)}{a_{-}-a_{0}-2 K \nu(\lambda)}\right] \rho\left(p_{\lambda}^{\varepsilon}, p_{\lambda}^{\varepsilon_{0}}\right) \\
& \quad+\left[\frac{4 K^{2}}{a_{-}-a_{0}-4 K \nu(\lambda)}+\frac{4 K^{2}}{a_{-}-a_{0}-2 K \nu(\lambda)}\right] C_{1}(\varepsilon) .
\end{aligned}
$$

Therefore

$$
\begin{aligned}
\rho\left(p_{\lambda}^{\varepsilon}, p_{\lambda}^{\varepsilon_{0}}\right) & \leq\left[\frac{2 K^{2} \nu(\lambda)}{a_{-}-a_{0}-4 K \nu(\lambda)}+\frac{2 K^{2} \nu(\lambda)}{a_{-}-a_{0}-2 K \nu(\lambda)}\right] \rho\left(p_{\lambda}^{\varepsilon}, p_{\lambda}^{\varepsilon_{0}}\right) \\
& +\left[\frac{4 K^{2}}{a_{-}-a_{0}-4 K \nu(\lambda)}+\frac{4 K^{2}}{a_{-}-a_{0}-2 K \nu(\lambda)}\right] C_{1}(\varepsilon) .
\end{aligned}
$$

Choosing $\lambda$ sufficiently small, we have

$$
\left[\frac{2 K^{2} \nu(\lambda)}{a_{-}-a_{0}-4 K \nu(\lambda)}+\frac{2 K^{2} \nu(\lambda)}{a_{-}-a_{0}-2 K \nu(\lambda)}\right]<\frac{1}{2} .
$$


Thus

$$
\rho\left(p_{\lambda}^{\varepsilon}, p_{\lambda}^{\varepsilon_{0}}\right)<\frac{1}{2} \rho\left(p_{\lambda}^{\varepsilon}, p_{\lambda}^{\varepsilon_{0}}\right)+C_{2}(\varepsilon)
$$

where $C_{2}(\varepsilon)=\left[\frac{4 K^{2}}{a_{-}-a_{0}-4 K \nu(\lambda)}+\frac{4 K^{2}}{a_{-}-a_{0}-2 K \nu(\lambda)}\right] C_{1}(\varepsilon)$.

Therefore

$$
\rho\left(p_{\lambda}^{\varepsilon}, p_{\lambda}^{\varepsilon_{0}}\right)<2 C_{2}(\varepsilon),
$$

where $C_{2}(\varepsilon) \rightarrow 0$, as $\varepsilon \rightarrow \varepsilon_{0}$, concluding the proof.

3.2. Continuity of the center-stable (center-unstable) manifolds. In this section we prove the continuity of the center-stable and centerunstable manifolds.

Theorem 3.2. Assume the same hypotheses of Theorem 3.1. Then the center stable, center unstable manifolds, $W_{\varepsilon}^{* s}, W_{\varepsilon}^{* u}$ are continuous at $\varepsilon_{0}$.

Proof We prove the continuity of $W_{\varepsilon}^{* s}$. The continuity of $W_{\varepsilon}^{* u}$ follows in a similar way. By Theorem 2.6, $W_{\varepsilon}^{* s}$ is the graph of a Lipschitz function $p_{\lambda}^{* \varepsilon}$, where $p_{\lambda}^{* \varepsilon}$ satisfies

$$
\begin{aligned}
& w_{-}(t, \varepsilon)=T(t) \varphi_{-}+\int_{0}^{t} T(t-s) \pi_{-} f_{\lambda}^{\varepsilon}\left(w_{-}(s, \varepsilon)+w_{0}(s, \varepsilon)\right. \\
&\left.+p_{\lambda}^{* \varepsilon}\left(w_{-}(s, \varepsilon)+w_{0}(s, \varepsilon)\right)\right) d s \\
& w_{0}(t, \varepsilon)=T(t) \varphi_{0}+\int_{0}^{t} T(t-s) \pi_{0} f_{\lambda}^{\varepsilon}\left(w_{-}(s, \varepsilon)+w_{0}(s, \varepsilon)\right. \\
&\left.+p_{\lambda}^{* \varepsilon}\left(w_{-}(s, \varepsilon)+w_{0}(s, \varepsilon)\right)\right) d s \\
& p_{\lambda}^{* \varepsilon}\left(\varphi_{-}+\varphi_{0}\right)=\int_{\infty}^{0} T(-s) \pi_{+} f_{\lambda}^{\varepsilon}\left(w_{-}(s, \varepsilon)\right.+w_{0}(s, \varepsilon) \\
&\left.+p_{\lambda}^{* \varepsilon}\left(w_{-}(s, \varepsilon)+w_{0}(s, \varepsilon)\right)\right) d s
\end{aligned}
$$

Furthermore, $p_{\lambda}^{*}$ has Lipschitz constant equal or smaller than 1 and $p_{\lambda}^{*}(0)=0$.

To facilitate the notation, from now on, in this subsection, we write $p_{\lambda}^{\varepsilon}$ in the place of $p_{\lambda}^{* \varepsilon}$. 
Using (3.10), we have

$$
\begin{aligned}
\left\|w_{-}(s, \varepsilon)+w_{0}(s, \varepsilon)\right\| & \leq K e^{-a_{-} t}\left\|\varphi_{-}\right\|+\int_{0}^{t} K \nu(\lambda) e^{-(t-s) a_{-}} \| w_{-}(s, \varepsilon) \\
& +w_{0}(s, \varepsilon)+p_{\lambda}^{\varepsilon}\left(w_{-}(s, \varepsilon)+w_{0}(s, \varepsilon)\right) \| d s \\
& +K e^{a_{0} t}\left\|\varphi_{0}\right\|+\int_{0}^{t} K \nu(\lambda) e^{(t-s) a_{0}} \| w_{-}(s, \varepsilon)+w_{0}(s, \varepsilon) \\
& +p_{\lambda}^{\varepsilon}\left(w_{-}(s, \varepsilon)+w_{0}(s, \varepsilon)\right) \| d s \\
& \leq K e^{-a_{-} t}\left\|\varphi_{-}\right\|+\int_{0}^{t} 2 K \nu(\lambda) e^{-(t-s) a_{-}} \| w_{-}(s, \varepsilon) \\
& +w_{0}(s, \varepsilon) \| d s \\
& +K e^{a_{0} t}\left\|\varphi_{0}\right\|+\int_{0}^{t} 2 K \nu(\lambda) e^{(t-s) a_{0}} \| w_{-}(s, \varepsilon) \\
& +w_{0}(s, \varepsilon) \| d s
\end{aligned}
$$

Since $-a_{-}<a_{0}$, it follows that

$$
\begin{aligned}
& \left\|w_{-}(s, \varepsilon)+w_{0}(s, \varepsilon)\right\| \\
& \leq K e^{a_{0} t}\left\|\varphi_{0}\right\|+\int_{0}^{t} 4 K \nu(\lambda) e^{(t-s) a_{0}}\left\|w_{-}(s, \varepsilon)+w_{0}(s, \varepsilon)\right\| d s .
\end{aligned}
$$

By Gronwall's Lemma, we obtain

$$
\left\|w_{-}(s, \varepsilon)+w_{0}(s, \varepsilon)\right\| \leq K\left\|\varphi_{-}+\varphi_{0}\right\| e^{\left(4 K \nu(\lambda)+a_{0}\right) t} .
$$

Let $\rho^{*}$ be the metric given by

$$
\rho^{*}\left(h_{1}, h_{2}\right)=\sup _{\varphi \in X, \varphi_{-}+\varphi_{0} \neq 0} \frac{\left\|h_{1}\left(\varphi_{-}+\varphi_{0}\right)-h_{2}\left(\varphi_{-}+\varphi_{0}\right)\right\|}{\left\|\varphi_{-}+\varphi_{0}\right\|}
$$

equipped with which, the set

$$
\begin{gathered}
G^{*}=\left\{h: \pi_{-} X \oplus \pi_{0} X \rightarrow \pi_{+} X \oplus \pi_{0} X,\left\|h\left(\varphi_{-}+\varphi_{0}\right)-h\left(\psi_{+}+\psi_{0}\right)\right\|\right. \\
\left.\leq\left\|\varphi_{-}+\varphi_{0}-\psi_{-}+\psi_{0}\right\|, \forall \varphi, \psi \in X, h(0)=0\right\}
\end{gathered}
$$


becomes a complete metric space. Let $\theta^{*}(t)=\| w_{-}(t, \varepsilon)+w_{0}(t, \varepsilon)-$ $w_{-}\left(t, \varepsilon_{0}\right)-w_{0}\left(t, \varepsilon_{0}\right) \mid, t \geq 0$. Then

$$
\begin{aligned}
\theta^{*}(t) & \leq \int_{0}^{t} K e^{-(t-s) a_{-}} \| f_{\lambda}^{\varepsilon}\left[w_{-}(s, \varepsilon)+w_{0}(s, \varepsilon)+p_{\lambda}^{\varepsilon}\left(w_{-}(s, \varepsilon)+w_{0}(s, \varepsilon)\right)\right] \\
& -f_{\lambda}^{\varepsilon_{0}}\left[w_{-}\left(s, \varepsilon_{0}\right)+w_{0}\left(s, \varepsilon_{0}\right)+p_{\lambda}^{\varepsilon_{0}}\left(w_{-}\left(s, \varepsilon_{0}\right)+w_{0}\left(s, \varepsilon_{0}\right)\right)\right] \| d s \\
& +\int_{0}^{t} K e^{(t-s) a_{0}} \| f_{\lambda}^{\varepsilon}\left[w_{-}(s, \varepsilon)+w_{0}(s, \varepsilon)+p_{\lambda}^{\varepsilon}\left(w_{-}(s, \varepsilon)+w_{0}(s, \varepsilon)\right)\right] \\
& -f_{\lambda}^{\varepsilon_{0}}\left[w_{-}\left(s, \varepsilon_{0}\right)+w_{0}\left(s, \varepsilon_{0}\right)+p_{\lambda}^{\varepsilon_{0}}\left(w_{-}\left(s, \varepsilon_{0}\right)+w_{0}\left(s, \varepsilon_{0}\right)\right)\right] \| d s
\end{aligned}
$$

Using that $-(t-s) a_{-} \leq(t-s) a_{0}$, we obtain

$$
\begin{aligned}
\theta^{*}(t) & \leq \int_{0}^{t} 2 K e^{(t-s) a_{0}} \| f_{\lambda}^{\varepsilon}\left[w_{-}(s, \varepsilon)+w_{0}(s, \varepsilon)+p_{\lambda}^{\varepsilon}\left(w_{-}(s, \varepsilon)+w_{0}(s, \varepsilon)\right)\right] \\
& -f_{\lambda}^{\varepsilon_{0}}\left[w_{-}\left(s, \varepsilon_{0}\right)+w_{0}\left(s, \varepsilon_{0}\right)+p_{\lambda}^{\varepsilon_{0}}\left(w_{-}\left(s, \varepsilon_{0}\right)+w_{0}\left(s, \varepsilon_{0}\right)\right)\right] \| d s
\end{aligned}
$$

Summing and subtracting the term

$$
f_{\lambda}^{\varepsilon}\left[w_{-}\left(s, \varepsilon_{0}\right)+w_{0}\left(s, \varepsilon_{0}\right)+p_{\lambda}^{\varepsilon_{0}}\left(w_{-}\left(s, \varepsilon_{0}\right)+w_{0}\left(s, \varepsilon_{0}\right)\right)\right],
$$

and using (3.9), (3.10), we have

$$
\begin{aligned}
\theta^{*}(t) & \leq \int_{0}^{t} 2 K e^{(t-s) a_{0}} \nu(\lambda)\left[\theta^{*}(s)+\| p_{\lambda}^{\varepsilon}\left(w_{-}(s, \varepsilon)+w_{0}(s, \varepsilon)\right)\right. \\
& \left.-p_{\lambda}^{\varepsilon_{0}}\left(w_{-}\left(s, \varepsilon_{0}\right)+w_{0}\left(s, \varepsilon_{0}\right)\right) \|\right] d s \\
& +\int_{0}^{t} 2 K e^{(t-s) a_{0}} C_{1}(\varepsilon)\left\|w_{-}\left(s, \varepsilon_{0}\right)+w_{0}\left(s, \varepsilon_{0}\right)\right\| d s .
\end{aligned}
$$

Now, using that $p_{\lambda}^{\varepsilon}$ is Lipschitzian with Lipschitz constant smaller than 1 and $p_{\lambda}^{\varepsilon_{0}}(0)=0$, it follows that

$$
\begin{aligned}
& \left\|p_{\lambda}^{\varepsilon}\left(w_{-}(s, \varepsilon)+w_{0}(s, \varepsilon)\right)-p_{\lambda}^{\varepsilon_{0}}\left(w_{-}\left(s, \varepsilon_{0}\right)+w_{0}\left(s, \varepsilon_{0}\right)\right)\right\| \\
& \leq\left\|w_{-}(s, \varepsilon)+w_{0}(s, \varepsilon)-w_{-}\left(s, \varepsilon_{0}\right)-w_{0}\left(s, \varepsilon_{0}\right)\right\|+\| w_{-}\left(s, \varepsilon_{0}\right) \\
& -w_{0}\left(s, \varepsilon_{0}\right) \| \frac{\left\|p_{\lambda}^{\varepsilon}\left(w_{-}\left(s, \varepsilon_{0}\right)+w_{0}\left(s, \varepsilon_{0}\right)\right)-p_{\lambda}^{\varepsilon_{0}}\left(w_{-}\left(s, \varepsilon_{0}\right)+w_{0}\left(s, \varepsilon_{0}\right)\right)\right\|}{\left\|w_{-}\left(s, \varepsilon_{0}\right)-w_{0}\left(s, \varepsilon_{0}\right)\right\|} \\
& \leq \theta^{*}(s)+\rho^{*}\left(p_{\lambda}^{\varepsilon}, p_{\lambda}^{\varepsilon_{0}}\right)\left\|w_{-}\left(s, \varepsilon_{0}\right)+w_{0}\left(s, \varepsilon_{0}\right)\right\| .
\end{aligned}
$$

Then

$$
\begin{aligned}
\theta^{*}(t) & \leq \int_{0}^{t} 2 K e^{(t-s) a_{0}} \nu(\lambda)\left[2 \theta^{*}(s)+\left\|w_{-}\left(s, \varepsilon_{0}\right)+w_{0}\left(s, \varepsilon_{0}\right)\right\| \rho^{*}\left(p_{\lambda}^{\varepsilon}, p_{\lambda}^{\varepsilon_{0}}\right)\right] d s \\
& +\int_{0}^{t} 2 K C_{1}(\varepsilon) e^{(t-s) a_{0}}\left\|w_{-}\left(s, \varepsilon_{0}\right)+w_{0}\left(s, \varepsilon_{0}\right)\right\| d s
\end{aligned}
$$


Using (3.13), we obtain

$$
\begin{aligned}
\theta^{*}(t) & \leq \int_{0}^{t} 4 K \nu(\lambda) e^{(t-s) a_{0}} \theta^{*}(s) d s \\
& +\int_{0}^{t} 2 K e^{(t-s) a_{0}} \nu(\lambda) K\left\|\varphi_{-}+\varphi_{0}\right\| e^{\left(4 K \nu(\lambda)+a_{0}\right) s} d s \rho^{*}\left(p_{\lambda}^{\varepsilon}, p_{\lambda}^{\varepsilon_{0}}\right) d s \\
& +\int_{0}^{t} 2 K C_{1}(\varepsilon) e^{(t-s) a_{0}} K\left\|\varphi_{-}+\varphi_{0}\right\| e^{\left(4 K \nu(\lambda)+a_{0}\right) s} d s .
\end{aligned}
$$

Thus

$$
\begin{aligned}
e^{-a_{0} t} \theta^{*}(t) & \leq \int_{0}^{t} 4 K \nu(\lambda) e^{-a_{0} s} \theta^{*}(s) d s \\
& +2 K^{2} \nu(\lambda)\left\|\varphi_{-}+\varphi_{0}\right\| \rho^{*}\left(p_{\lambda}^{\varepsilon}, p_{\lambda}^{\varepsilon_{0}}\right) \int_{0}^{t} e^{4 K \nu(\lambda) s} d s \\
& +2 K^{2} C_{1}(\varepsilon)\left\|\varphi_{-}+\varphi_{0}\right\| \int_{0}^{t} e^{4 K \nu(\lambda) s} d s .
\end{aligned}
$$

Since

$$
\int_{0}^{t} e^{4 K \nu(\lambda) s} d s \leq \frac{e^{4 K \nu(\lambda) t}}{4 K \nu(\lambda)}
$$

we obtain

$$
\begin{aligned}
e^{-a_{0} t} \theta^{*}(t) & \leq \int_{0}^{t} 4 K \nu(\lambda) e^{-a_{0} s} \theta^{*}(s) d s+\frac{K}{2}\left\|\varphi_{-}+\varphi_{0}\right\| \rho^{*}\left(p_{\lambda}^{\varepsilon}, p_{\lambda}^{\varepsilon_{0}}\right) e^{4 K \nu(\lambda) t} \\
& +\frac{K}{2 \nu(\lambda)} C_{1}(\varepsilon)\left\|\varphi_{-}+\varphi_{0}\right\| e^{4 K \nu(\lambda) t}
\end{aligned}
$$

From Gronwall's Lemma, it follows that

$$
\theta^{*}(t) \leq\left[\frac{K}{2} \rho^{*}\left(p_{\lambda}^{\varepsilon}, p_{\lambda}^{\varepsilon_{0}}\right)+\frac{K}{2 \nu(\lambda)} C_{1}(\varepsilon)\right] e^{\left(a_{0}+8 K \nu(\lambda)\right) t}\left\|\varphi_{-}+\varphi_{0}\right\| .
$$

Now

$$
\begin{aligned}
\left\|p_{\lambda}^{\varepsilon}\left(\varphi_{-}+\varphi_{0}\right)-p_{\lambda}^{\varepsilon_{0}}\left(\varphi_{-}+\varphi_{0}\right)\right\| & \leq \int_{0}^{\infty} K e^{-a_{+} s} \| f_{\lambda}^{\varepsilon}\left(w_{-}(s, \varepsilon)+w_{0}(s, \varepsilon)\right. \\
& \left.+p_{\lambda}^{\varepsilon}\left(w_{-}(s, \varepsilon)+w_{0}(s, \varepsilon)\right)\right) \\
& -f_{\lambda}^{\varepsilon_{0}}\left(w_{-}\left(s, \varepsilon_{0}\right)+w_{0}\left(s, \varepsilon_{0}\right)\right. \\
& \left.+p_{\lambda}^{\varepsilon_{0}}\left(w_{-}\left(s, \varepsilon_{0}\right)+w_{0}\left(s, \varepsilon_{0}\right)\right)\right) \| d s .
\end{aligned}
$$

Using (3.9) and (3.10), after adding and subtracting the term

$$
f_{\lambda}^{\varepsilon}\left(w_{-}\left(s, \varepsilon_{0}\right)+w_{0}\left(s, \varepsilon_{0}\right)+p_{\lambda}^{\varepsilon_{0}}\left(w_{-}\left(s, \varepsilon_{0}\right)+w_{0}\left(s, \varepsilon_{0}\right)\right)\right),
$$


we have

$$
\begin{aligned}
\left\|p_{\lambda}^{\varepsilon}\left(\varphi_{-}+\varphi_{0}\right)-p_{\lambda}^{\varepsilon_{0}}\left(\varphi_{-}+\varphi_{0}\right)\right\| & \leq \int_{0}^{\infty} K \nu(\lambda) e^{-a_{+} s} \theta^{*}(s) d s \\
& +\int_{0}^{\infty} K \nu(\lambda) e^{-a_{+} s} \| p_{\lambda}^{\varepsilon}\left(w_{-}(s, \varepsilon)+w_{0}(s, \varepsilon)\right) \\
& -p_{\lambda}^{\varepsilon_{0}}\left(w_{-}\left(s, \varepsilon_{0}\right)+w_{0}\left(s, \varepsilon_{0}\right)\right) \| d s \\
& +\int_{0}^{\infty} K e^{-a_{+} s} C_{1}(\varepsilon) \| w_{-}\left(s, \varepsilon_{0}\right)+w_{0}\left(s, \varepsilon_{0}\right) \\
& +p_{\lambda}^{\varepsilon_{0}}\left(w_{-}\left(s, \varepsilon_{0}\right)+w_{0}\left(s, \varepsilon_{0}\right)\right) \| d s
\end{aligned}
$$

Using that

$$
\begin{aligned}
\| p_{\lambda}^{\varepsilon}\left(w_{-}(s, \varepsilon)+w_{0}(s, \varepsilon)\right)- & p_{\lambda}^{\varepsilon_{0}}\left(w_{-}\left(s, \varepsilon_{0}\right)+w_{0}\left(s, \varepsilon_{0}\right)\right) \| \\
& \leq \theta^{*}(s)+\rho^{*}\left(p_{\lambda}^{\varepsilon}, p_{\lambda}^{\varepsilon_{0}}\right)\left\|w_{-}\left(s, \varepsilon_{0}\right)+w_{0}\left(s, \varepsilon_{0}\right)\right\|
\end{aligned}
$$

we obtain

$$
\begin{aligned}
& \left\|p_{\lambda}^{\varepsilon}\left(\varphi_{-}+\varphi_{0}\right)-p_{\lambda}^{\varepsilon_{0}}\left(\varphi_{-}+\varphi_{0}\right)\right\| \\
& \leq \int_{0}^{\infty} 2 K \nu(\lambda) e^{-a_{+} s} \theta^{*}(s) d s+\int_{0}^{\infty} K \nu(\lambda) e^{-a_{+} s} \rho^{*}\left(p_{\lambda}^{\varepsilon}, p_{\lambda}^{\varepsilon_{0}}\right) \| w_{-}\left(s, \varepsilon_{0}\right) \\
& +w_{0}\left(s, \varepsilon_{0}\right)\left\|d s+\int_{0}^{\infty} 2 K C_{1}(\varepsilon) e^{-a_{+} s}\right\| w_{-}\left(s, \varepsilon_{0}\right)+w_{0}\left(s, \varepsilon_{0}\right) \| d s
\end{aligned}
$$


Using (3.13) and (3.14), it follows that

$$
\begin{aligned}
& \left\|p_{\lambda}^{\varepsilon}\left(\varphi_{-}+\varphi_{0}\right)-p_{\lambda}^{\varepsilon_{0}}\left(\varphi_{-}+\varphi_{0}\right)\right\| \\
& \leq\left(K^{2} \nu(\lambda)\left\|\varphi_{-}+\varphi_{0}\right\| \int_{0}^{\infty} e^{-\left(a_{+}-a_{0}-8 K \nu(\lambda)\right) s} d s\right) \rho^{*}\left(p_{\lambda}^{\varepsilon}, p_{\lambda}^{\varepsilon_{0}}\right) \\
& +\left(K^{2} \nu(\lambda)\left\|\varphi_{-}+\varphi_{0}\right\| \int_{0}^{\infty} e^{-\left(a_{+}-a_{0}-4 K \nu(\lambda)\right) s} d s\right) \rho^{*}\left(p_{\lambda}^{\varepsilon}, p_{\lambda}^{\varepsilon_{0}}\right) \\
& +\left(K^{2}\left\|\varphi_{-}+\varphi_{0}\right\| \int_{0}^{\infty} e^{-\left(a_{+}-a_{0}-8 K \nu(\lambda)\right) s} d s\right) C_{1}(\varepsilon) \\
& +\left(2 K\left\|\varphi_{-}+\varphi_{0}\right\| \int_{0}^{\infty} e^{-\left(a_{+}-a_{0}-4 K \nu(\lambda)\right) s} d s\right) C_{1}(\varepsilon) \\
& =\left[\frac{K^{2} \nu(\lambda)}{a_{+}-a_{0}-8 K \nu(\lambda)}\left\|\varphi_{-}+\varphi_{0}\right\|\right. \\
& \left.+\frac{K^{2} \nu(\lambda)}{a_{+}-a_{0}-4 K \nu(\lambda)}\left\|\varphi_{-}+\varphi_{0}\right\|\right] \rho^{*}\left(p_{\lambda}^{\varepsilon}, p_{\lambda}^{\varepsilon_{0}}\right) \\
& +\left[\frac{K^{2}}{a_{+}-a_{0}-8 K \nu(\lambda)}\left\|\varphi_{-}+\varphi_{0}\right\|+\frac{2 K}{a_{+}-a_{0}-4 K \nu(\lambda)}\left\|\varphi_{-}+\varphi_{0}\right\|\right] C_{1}(\varepsilon) .
\end{aligned}
$$

\section{Hence}

$$
\begin{aligned}
& \frac{\left\|p_{\lambda}^{\varepsilon}\left(\varphi_{-}+\varphi_{0}\right)-p_{\lambda}^{\varepsilon_{0}}\left(\varphi_{-}+\varphi_{0}\right)\right\|}{\left\|\varphi_{-}+\varphi_{0}\right\|} \\
& \leq\left[\frac{K^{2} \nu(\lambda)}{a_{+}-a_{0}-8 K \nu(\lambda)}+\frac{K^{2} \nu(\lambda)}{a_{+}-a_{0}-4 K \nu(\lambda)}\right] \rho^{*}\left(p_{\lambda}^{\varepsilon}, p_{\lambda}^{\varepsilon_{0}}\right) \\
& \quad+\left[\frac{K^{2}}{a_{+}-a_{0}-8 K \nu(\lambda)}+\frac{2 K}{a_{+}-a_{0}-4 K \nu(\lambda)}\right] C_{1}(\varepsilon) .
\end{aligned}
$$

Choosing $\lambda$ small enough as to have

$$
\left[\frac{K^{2} \nu(\lambda)}{a_{+}-a_{0}-8 K \nu(\lambda)}+\frac{K^{2} \nu(\lambda)}{a_{+}-a_{0}-4 K \nu(\lambda)}\right]<\frac{1}{2}
$$

and letting

$$
C_{2}^{*}(\varepsilon)=\left[\frac{K^{2}}{a_{+}-a_{0}-8 K \nu(\lambda)}+\frac{2 K}{a_{+}-a_{0}-4 K \nu(\lambda)}\right] C_{1}(\varepsilon),
$$

we obtain

$$
\rho^{*}\left(p_{\lambda}^{\varepsilon}, p_{\lambda}^{\varepsilon_{0}}\right)<\frac{1}{2} \rho^{*}\left(p_{\lambda}^{\varepsilon}, p_{\lambda}^{\varepsilon_{0}}\right)+C_{2}^{*}(\varepsilon) .
$$

Therefore

$$
\rho^{*}\left(p_{\lambda}^{\varepsilon}, p_{\lambda}^{\varepsilon_{0}}\right)<2 C_{2}^{*}(\varepsilon)
$$


where $C_{2}^{*}(\varepsilon) \rightarrow 0$, as $\varepsilon \rightarrow \varepsilon_{0}$, concluding the proof.

Remark 3.3. It follows from [15] that the center manifold, $W_{\varepsilon}^{c}$ is given by

$$
W_{\varepsilon}^{c}=W_{\varepsilon}^{* s} \cap W_{\varepsilon}^{* u} .
$$

Therefore, the the continuity of the center manifold, $W_{\varepsilon}^{c}$ also follows from Theorem 3.2.

\section{Acknowledgments}

The authors would like to thank the anonymous referee for his / her reading of the manuscript. The first author would also like to thank his daughter Luana for your understanding during the development of part this work.

\section{References}

[1] J. M. Arrieta, A. N. Carvalho; Spectral convergence and nonlinear dynamics of reaction-diffusion equations under perturbations of domain. J. Differential Equations 199, (2004), 143-178.

[2] J. Ball; Saddle point analysis for an ordinary differential equation in Banach space and application to buckling of a beam. Nonlinear Elasticity (R. W. Dickey, ed.) Academic Press, (1973), 937-948.

[3] L. Barreira and C. Valls;Smooth invariant manifolds in Banach spaces with nonuniform exponential dichotomy. J. Funct. Anal. 238 (2006) 118-148.

[4] L. Barreira and C. Valls; Parameter dependence of stable manifolds under nonuniform hyperbolicity. J. Math. Anal. Appl. 358 (2009) 419426.

[5] P. W. Bates, K. Lu and C. Zeng; Existence and Persistence of Invariant Manifolds for Semiflows in Banach Space. Memoirs of the American Mathematical Society, Vol. 135, No. 645. American Mathematical Society, 1998.

[6] J. Carr; Application of center manifold theory. Applied Mathematical Sciences N. 35, Springer-Verlag, 1981.

[7] S. N. Chow and K. Lu; Invariant manifolds for flows in Banach spaces. Journal of Differential Equations, Vol. 74, (1988), 285-317.

[8] S. N. Chow and K. Lu; $C^{k}$ centre unstable manifolds .Proceedings of the Royal Society of Edinburgh, Vol. 108A, (1988), 303-320.

[9] J. K. Daleckii and M. G. Krein; Stability of Solutions of Differential Equations in Banach Space. American Mathematical Society Providence, Rhode Island, 1974.

[10] N. Fenichel; Persistence and smoothness of invariant manifolds for flows. Indiana University Mathematics Journal, vol. 21, No. 3 (1971), 193-256.

[11] J. K. Hale and G. Raugel; Convergence in gradient-like systems with applications to PDE. ZAMP 43 (1992), 63-124 193-256.

[12] J. K. Hale; Ordinary Differential Equations. Pure and Applied Mathematics A Series of Texts and Monographs, No. XXI, 1980.

[13] D. Henry; Geometric Theory of Semilinear Parabolic Equations. Lecture Notes in Mathematics N. 840, Springer-Verlag, 1981.

[14] M. W. Hirsch, C. C. Pugh and M. Shub; Invariant Manifolds. Lecture Notes in Mathematics N. 583, Springer-Verlag, 1977. 
[15] A. Kelley; The stable, center-stable, center, center-unstable, unstable manifolds. J. Diff. Equations 3, (1976), 546-570.

[16] A. L. Pereira and M. C. Pereira; Continuity of attractors for a reaction-diffusion problem with nonlinear bounday conditions with respect to variations of the domain, Journal of Differential Equations, v. 239 , ( 2007), p. 343-370.

[17] A. L. Pereira, L. A. F. Oliveira; Invariant manifolds and limiting equations for a hyperbolic problem, Dynamics of Continuous, Discrete and Impulsive Systems, No. 7,(2000), p. 503-524.

[18] S. H. da Silva; Existência e continuidade de atrator global para uma equação de evolução com convolução, $\mathrm{PhD}$ Thesis, Instituto de Matemática e Estatística da Universidade de São Paulo, Brazil, 2007. 